\title{
Relations entre matière sèche à la floraison et rendement en grains chez le maïs : importance du rayonnement disponible par plante
}

\author{
F. Ruget \\ INRA, Station de Bioclimatologie, 78750 Thiverval-Grignon, France
}

(reçu le 8 juin 1988, accepté le 28 février 1989)

Résumé - Des indicateurs précoces de rendement sont souvent utilisés, mais la bonne connaissance de leur signification physiologique est essentielle à leur bon emploi. Ainsi en est-il du poids de matière sèche à la floraison.

Le présent travail montre que des plantes ayant des poids de matière sèche différents à la floraison peuvent produire la même quantité de grains. II s'agit de cultures à deux densités différentes ( $d$ et $D=2 d$ ). Le traitement le plus dense subit un éclaircissage légèrement antérieur à la floraison, le ramenant à une densité é proche de $\mathrm{d}$. Ni le nombre de fleurs initiées, ni le nombre de grains potentiels ne sont différents entre les deux cultures. Les plantes disposent donc après éclaircissage des mêmes quantités de rayonnement.

Les productions de grains par plante sont équivalentes. Les différences de production antérieures à l'éclaircissage se retrouvent à la récolte essentiellement sur le diamètre au collet des tiges et sur leur poids; le nombre de plantes sans épi est un peu plus élevé pour le traitement ayant subi une contrainte en début de cycle. A la période de contrainte la plus forte, c'est-à-dire juste avant l'éclaircissage, ces grandeurs étaient donc les seules sensibles.

Le poids de matière sèche à la floraison doit ses qualités de bon indicateur de la production à la récolte : - d'une part, à la relation entre surface foliaire et poids de matière sèche à la floraison,

- d'autre part, à la régularité de l'évolution de la surface foliaire après la floraison. En réalité, c'est le rayonnement disponible par plante après la floraison qui est déterminant pour la production de grains, dans nos conditions.

Les variabilités génétiques et climatiques de ces relations seront analysées dans des travaux ultérieurs.

maïs - floraison - rendement en grains - indicateur précoce - stockage temporaire de réserves

Summary - Relationship between dry matter at flowering stage and grain yield for maize: importance of available solar radiation for the plant. Many indices are used to estimate at an early stage (e.g. dry matter production at flowering) the final grain production. A knowledge of the physiological basis of these relations is a condition for their good use.

The present work shows that plants with different dry matter production at flowering can produce the same quantity of grains per plant. Plants were grown at two sowing densities ( $d$ and $D=2 d$ ) and a part of the thickest crop was partial$1 y$ thinned to a half density $(e=d)$ some days before flowering. Differentiated flower number and potential grain number were not different for the two crops, plants received the same radiation quantities after flowering, but they did not have the same dry weight at flowering.

Dry weight differences between crops at flowering are found at harvest essentially on stem weight, but grain production is not affected. Only basal stem diameter, stem weight and number of plants without ear (greater for stressed crop) show sensitivity at maximum stress period (days before thinning).

So the first factor for grain filling is the available global radiation per plant, if the potential grain number is not modified. Relations between dry matter at flowering and grain yield are based on a good leaf area ratio (leaf area/dry matter) and stability of plant leaf area after flowering. Dry weight at flowering is a good index for grain production if the amount of available radiation per plant remains the same.

Genotypic and environmental variability of these relations will be analyzed in a further study.

maize - flowering stage - grain yield - early stage estimation

\section{Introduction}

De nombreux utilisateurs sont intéressés par la possibilité d'estimer précocement des rendements en grains, que ce soit pour sélectionner des génotypes ou prévoir des productions agricoles; il existe donc de nombreux travaux portant sur les relations entre des critères biologiques mesurés précocement (matière sèche ou photosynthèse, à la floraison ou avant) et rendement 
en grains (Fuchs, 1977; Edmeades et Daynard, 1979b; Hawkins et Cooper, 1981). La signification de ces relations est différente selon qu'elles sont mises en évidence par comparaison de génotypes, de plantes dans un couvert ou de conditions de culture. Même dans le cas où les corrélations mises en évidence dépendent de relations physiologiques internes, leur origine est rarement recherchée. Or ces effets peuvent être liés à différentes causes, qu'il s'agisse de niveau d'assimilation possible ultérieurement (persistance de l'indice foliaire), de quantité de réserves préalablement formées, de nombre de fleurs initiées ou fécondables.

L'indice foliaire détermine la quantité d'assimilats disponibles pour l'ensemble du couvert donc la croissance globale du couvert, tandis que la quantité disponible par plante, liée à la surface foliaire par plante, détermine la répartition entre organes : en dessous d'une certaine quantité d'assimilats disponibles, la plante ne remplit pas de grains (Edmeades et Daynard, 1979a).

Le rôle de réserve temporaire joué par la tige est bien connu (Daynard et al., 1969; Hanway et Russell, 1969; Jones et Simmons, 1983), mais la question se pose parfois d'une concurrence entre tige et épi (Tollenaar, 1977) : qu'en est-il ? Quand les réserves commencent-elles à se constituer? Peuvent-elles être limitées par des capacités physiques de stockage ou dépendent-elles essentiellement des assimilats disponibles?

Enfin, le nombre de fleurs initiées ne semble que rarement limitant (Duncan, 1975; Edmeades et Daynard, 1979a; Derieux et al., 1985), mais le gradient de différenciation qui s'établit le long de l'épi peut aussi expliquer d'importantes limitations de production (Landi et Concilio, 1982; Ruget, 1987).

Notre expérimentation consiste à produire des plantes ayant des poids de matière sèche différents, mais disposant après une date donnée de ressources en rayonnement analogues. La méthode utilisée est la comparaison de deux densités de culture : l'une est normale $(d=n)$, l'autre d'abord forte (environ le double : $D=2 n$ ) est éclaircie de façon à revenir à la densité normale (donc le traitement éclairci (é) est $2 n$ puis n). La date choisie pour l'éclaircissage se situe quelques jours avant la floraison.

\section{Matériel et Méthodes}

\section{Génotype utilisé : Dea}

Conditions de culture : au champ, à Mons-en-Chaussée (Somme, $50^{\circ} \mathrm{N}, 3^{\circ} \mathrm{E}$ ), en 1984; date de semis : 4 mai.

Traitements : culture en densité faible (d), proche de la "normale" (67 500 plantes/ha), et en densité forte $(D=120000$ plantes/ha), éclaircie en partie le 7 août, c'est-à-dire quelques jours avant la floraison pour revenir à une valeur proche de la densité faible (é=60 000 plantes/ha).

\section{Observations et mesures:}

1. Suivi de la croissance de la partie aérienne - mesures biologiques : matière sèche, hauteur et diamètre au collet,

- rythme d'observation : les plantes sont prélevées tous les 3 à 4 jours avant la floraison, toutes les semaines (à 2 semaines) après,

- échantillon : à chaque date, sont prélevées 6 plantes consécutives dans 3 zones de chaque parcelle. Elles sont observées individuellement sauf pour les pesées entre la floraison et les deux dernières dates avant la récolte, où elles sont groupées par lots de 3.

- séparation en organes :

d'abord, les pesées sont faites sur les plantes entières, jusqu'à ce que la tige commence à s'allonger $(>10 \mathrm{~cm})$,

puis les limites sont séparés de l'ensemble tige + gaines,

la panicule est séparée au niveau du dernier nœud dès qu'elle est visible,

puis l'ensemble de l'épi est séparé, lui-même dissocié ensuite en spathes + pédoncule et épi proprement dit,

enfin, lorsque la consistance des grains rend l'opération réalisable facilement, les grains sont séparés de la rafle.

2. Différenciation florale femelle : de l'initiation à la floraison, sont observés sur les mêmes lots de plantes,

le stade de la base de l'épi,

le nombre total d'étages de fleurs initiées, et le nombre d'étages de fleurs ayant dépassé le stade C4 (Ruget, 1987).

3. Surface foliaire : les longueurs et largeurs des feuilles sont mesurées après floraison, c'est-à-dire après que celles-ci ont atteint leur taille finale, et les surfaces sont calculées par le produit longueur $x$ largeur x 0,75 (Bonhomme et al., 1982).

Le nombre de feuilles encore vertes est noté le 11 octobre.

4. Composantes du rendement à la récolte : les nombres de grains par épi sont comptés sur les échantillons qui servent à déterminer les poids de matière sèche, tandis que les nombres d'épis par plante sont observés au champ sur de plus grands nombres de plantes non prélevées.

5. Efficience de la transformation du rayonnement solaire en matière sèche. Le rayonnement capté par la culture est calculé à partir de la formule de Bonhomme et al. (1982)

$$
0,46\left(1-e^{(-0,7 I F)}\right)
$$

où IF est l'indice foliaire, considéré ici comme constant de l'éclaircissage (jour 220) à quelques jours avant la récolte (jour 283), où l'accumulation de matière sèche semble s'arrêter.

\section{Résultats}

Les résultats sont présentés en deux sous-chapitres :

- l'état des 2 couverts à l'éclaircissage : caractéristiques de la croissance (matière sèche présen- 
te et importance des différents organes, surface foliaire maximale) et du développement (nombre potentiel de grains);

- l'état des cultures à la récolte, complété de quelques éléments sur l'évolution de la culture entre floraison et récolte.

\section{Etat des cultures à l'éclaircissage}

Caractéristiques de la croissance (Tableaux l et II)

Les poids de matière sèche par plante sont significativement différents; la proportion de tige par plante est également différente, tandis que les hauteurs de tige à l'éclaircissage et une semaine avant ne le sont pas (Tableau I).

Les vitesses de croissance pour la plante entière dans les semaines qui entourent l'éclaircissage (Tableau II) sont sensiblement différentes (rapport $d / \hat{e}=1,15$ ) et celles des tiges le sont dans le même rapport $(1,17)$, celles des épis le sont nettement plus (rapport d/é=1,29).

La comparaison des pentes et ordonnées à l'origine des régressions (Tableau II) ne fait pas apparaître de différences significatives pour les plantes entières, tandis qu'elles le sont pour les

Tableau I. Caractéristiques de croissance à l'éclaircissage.

\begin{tabular}{|c|c|c|c|c|c|c|c|c|c|}
\hline & \multirow[t]{2}{*}{ Date } & \multicolumn{2}{|c|}{ Densité d } & \multicolumn{2}{|c|}{ Densité é } & \multirow{2}{*}{$\begin{array}{l}\text { Nb de } \\
\text { mesures }\end{array}$} & \multirow{2}{*}{\multicolumn{3}{|c|}{$\begin{array}{c}\text { tà } 1 \% \\
\text { critère de comparaison }\end{array}$}} \\
\hline & & moy & e.-t. & moy & e.-t. & & & & \\
\hline Eclaircissage & $7 / 8$ & & & & & & & & \\
\hline Matière sèche & $6 / 8$ & & & & & & & & \\
\hline par plante $(\mathrm{g})$ & & 83,5 & & 58,9 & & & & & \\
\hline par lot de 3 plantes & & 250,5 & 20,3 & 176,7 & 21,3 & 6 & 6,14 & 4,03 & $\mathbf{S}$ \\
\hline Tige/plante entière (\%) & $6 / 8$ & 60,5 & 1,19 & 58,17 & 0,67 & 18 & 7,24 & 2,9 & $\mathrm{~S}$ \\
\hline \multirow[t]{2}{*}{ Hauteur de tige $(\mathrm{m})$} & $30 / 7$ & 0,799 & 0,08 & 0,787 & 0,074 & 18 & 0,47 & 2,9 & NS \\
\hline & $6 / 8$ & 1,328 & 0,133 & 1,365 & 0,047 & 5 & $-0,59$ & 4,6 & NS \\
\hline
\end{tabular}

Tableau II. Comparaison des vitesses de croissance des deux densités autour de l'éclaircissage (ces vitesses correspondent aux pentes des régressions: matière sèche/temps).

Croissance du 27 juillet au 13 août

$\begin{array}{lllll}\text { Pente Intervalle } & \begin{array}{l}\text { Ordonnée } \\ \text { de confiance }\end{array} & \begin{array}{l}\text { Intervalle } \\ \text { àlorigine }\end{array} & \begin{array}{l}\text { Coefficient } \\ \text { de confiance }\end{array} \\ & \text { de corrélation }\end{array}$

\begin{tabular}{llc}
$\begin{array}{l}\text { Densité d } \\
\text { Plante entière } \\
\quad \text { jours 209, 212, 219, 227) }\end{array}$ & 3,53 & 0,76 \\
$\begin{array}{l}\text { Tige } \\
\text { (jours 209, 212, 219, 227) }\end{array}$ & 2,17 & 0,70 \\
$\begin{array}{l}\text { Feuilles } \\
\quad \text { jours 209, 212, 219, 227) }\end{array}$ & 0,18 & 0,26 \\
$\begin{array}{l}\text { Spathes + rafle } \\
\text { (jours 219, 227, 234) }\end{array}$ & 1,34 & 0,11 \\
$\begin{array}{l}\text { Densité é } \\
\text { Plante entière }\end{array}$ & & \\
$\quad$ jours 209, 212, 219, 227) & 3,06 & 1,26 \\
$\begin{array}{l}\text { Tige } \\
\quad \text { jours 209, 212, 219, 227) }\end{array}$ & 1,86 & 0,73 \\
$\begin{array}{l}\text { Feuilles } \\
\text { (jours 209, 212, 219, 227) }\end{array}$ & 0,22 & 0,44 \\
$\begin{array}{l}\text { Spathes + rafle } \\
\quad \text { jours 219, 227, 234) }\end{array}$ & 1,04 & 0,13 \\
$\begin{array}{l}\text { Rapport d/é } \\
\text { Plante entière }\end{array}$ & $\begin{array}{l}\text { Rapport des pentes } \\
\text { Tige } \\
\text { Feuilles } \\
\text { Spathes + rafle }\end{array}$ & 1,15 \\
\hline
\end{tabular}


épis : cela signifie que date de début et vitesse de croissance des épis sont significativement différentes entre les 2 traitements.

Les croissances de feuilles sont très faibles dans les 2 cas, parce que celles-ci ont atteint leur poids maximal.

Les surfaces foliaires sont significativement différentes à leur valeur maximale.

\section{Caractéristiques du développement (Tableaux III et IV)}

Pour ce qui est de la différenciation florale, le stade de la base de l'épi est légèrement retardé en forte densité dès la fin du stade C4 (Tableau
Illa) mais ni le nombre total d'étages de fleurs initiées, ni le nombre potentiel d'étages de grains (observé à la fin du stade D) ne diffèrent significativement entre les deux traitements. Cela signifie que le gradient de différenciation des fleurs n'a pas été affecté par le traitement "densité» appliqué en début de végétation.

Le nombre potentiel d'étages de grains est sensiblement inférieur au nombre d'étages de fleurs, donc le nombre de fleurs lui-même n'est pas limitant (Tableau IV).

La floraison femelle (soies visibles sur la moitié des plantes) (Tableau IIlb) est sensiblement retardée pour la densité éclaircie (plus de 2

Tableau III. Caractéristiques du développement : a. différenciation florale (nombre d'individus à chaque stade). b. floraison (nombre de plantes à soies visibles).

a) Différenciation florale femelle (pour 18 plantes)

\begin{tabular}{|c|c|c|c|c|c|c|c|c|}
\hline Stade & $B$ & $C 1$ & C3 & $C 4$ & $D 1$ & $D 2$ & $D 3$ & E1 \\
\hline Date & \multicolumn{8}{|c|}{ Densité $d$} \\
\hline $10 / 7$ & 2 & 13 & 3 & & & & & \\
\hline $13 / 7$ & & & 6 & 12 & & & & \\
\hline $17 / 7$ & & & 1 & 17 & & & & \\
\hline $20 / 7$ & & & & 1 & 15 & 2 & & \\
\hline $24 / 7$ & & & & & & 3 & 15 & \\
\hline \multirow[t]{2}{*}{$27 / 7$} & & & & & & 2 & 11 & 5 \\
\hline & \multicolumn{8}{|c|}{ Densité é } \\
\hline $10 / 7$ & 1 & 13 & 4 & & & & & \\
\hline $13 / 7$ & & 3 & 6 & 9 & & & & \\
\hline $17 / 7$ & & & 2 & 15 & 1 & & & \\
\hline $20 / 7$ & & & & 1 & 16 & 1 & & \\
\hline $24 / 7$ & & & & & 1 & 5 & 12 & \\
\hline $27 / 7$ & & & & & & 1 & 16 & 1 \\
\hline \multicolumn{9}{|l|}{ b) Floraison } \\
\hline (pour 100 plantes) & \multicolumn{2}{|c|}{ Densité } & & & & & & \\
\hline Date & $d$ & $e ́$ & & & & & & \\
\hline $9 / 8$ & 33 & 14 & & & & & & \\
\hline $13 / 8$ & 73 & 59 & & & & & & \\
\hline $16 / 8$ & 89 & 89 & & & & & & \\
\hline
\end{tabular}

Tableau IV. Composantes du rendement à la floraison.

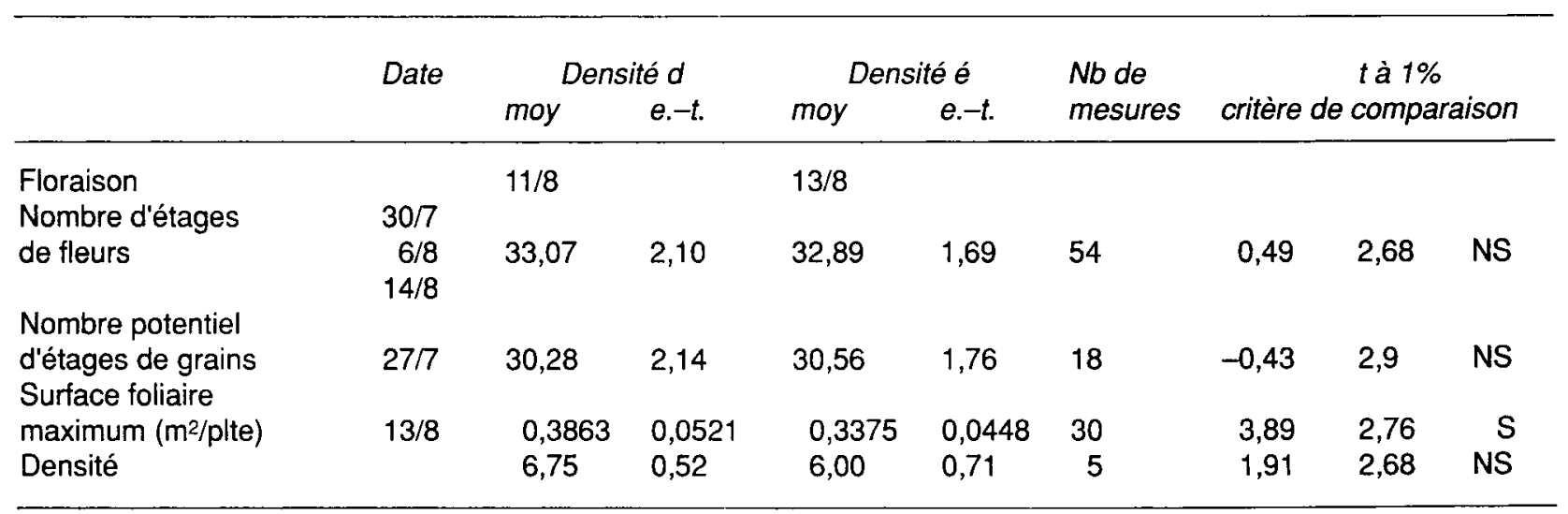


jours), mais il faut noter la date tardive de l'éclaircissage (moins de 4 jours avant la floraison de faible densité).

Croissance du couvert, de l'éclaircissage à la récolte et état des cultures à la récolte

Les productions sont d'abord présentées pour les plantes entières et par unité de surface de sol, pour comparer le fonctionnement global des 2 couverts après éclaircissage, puis par organe, de façon à situer l'effet des différences entre couverts acquises avant éclaircissage.
Production finale et croissance par plante et par unité de surface de sol (Tableau V)

Etant donné la faible variation de production entre les 2 dates de récolte, la croissance du couvert peut être considérée comme achevée dès la première date et les résultats des 2 dates comme répétitions.

Les quantités de matière sèche récoltées par unité de surface de sol sont nettement différentes, mais les croissances le sont peu.

L'analyse de variance des croissances par plante montre que celles-ci ne sont pas différentes entre traitements : il n'apparaît pas d'inter-

Tableau V. Production par plante et productivité par unité de surface de sol, après l'éclaircissage.

\begin{tabular}{|c|c|c|c|c|c|c|}
\hline & \multirow[t]{2}{*}{ Date } & & \multicolumn{2}{|c|}{ Densité $d=6,75$} & \multicolumn{2}{|c|}{ Densité é $=6,00$} \\
\hline & & & $\begin{array}{l}\text { par } \\
\text { plante } \\
(g)\end{array}$ & $\begin{array}{l}\text { par } \\
\text { unité de } \\
\text { surface } \\
\left(m^{2}\right)\end{array}$ & $\begin{array}{l}\text { par } \\
\text { plante } \\
(g)\end{array}$ & $\begin{array}{l}\text { par } \\
\text { unité de } \\
\text { surface } \\
\left(m^{2}\right)\end{array}$ \\
\hline \multicolumn{7}{|l|}{ Poids de matière sèche } \\
\hline éclaircissage & $6 / 8$ & 219 & 83,5 & 563,6 & 58,9 & 353,4 \\
\hline floraison & $13 / 8$ & 227 & 111,0 & 749,3 & 91,5 & 549,0 \\
\hline récolte & $9 / 10$ & 283 & 220,1 & 1485,7 & 193,1 & 1158,6 \\
\hline & $22 / 10$ & 296 & 207,5 & 1400,6 & 190,8 & 1144,8 \\
\hline \multicolumn{7}{|l|}{ croissance } \\
\hline écl.--récolte & $9 / 10$ & & 136,6 & 922,1 & 134,2 & 805,2 \\
\hline & $22 / 10$ & & 124,0 & 837,0 & 131,9 & 791,4 \\
\hline \multicolumn{7}{|l|}{ croissance } \\
\hline fl.-récolte & $9 / 10$ & & 109,1 & 736,4 & 101,6 & 609,6 \\
\hline & $22 / 10$ & & 96,5 & 651,4 & 99,3 & 595,8 \\
\hline Indice foliaire & & & & 2,61 & & 2,03 \\
\hline \multicolumn{7}{|l|}{ Proportion de } \\
\hline rayonnement capté & & & & 0,386 & & 0,349 \\
\hline \multicolumn{7}{|l|}{ Rayonnement global (MJ) } \\
\hline éclaircissage-récolte & & & & 692 & & 692 \\
\hline Rayonnement capté (MJ) & & & & 267,1 & & 241,5 \\
\hline \multicolumn{7}{|l|}{ Efficience de la } \\
\hline transformation $(\mathrm{g} / \mathrm{MJ})$ & $9 / 10$ & & & 3,45 & & 3,33 \\
\hline & $22 / 10$ & & & 3,13 & & 3,28 \\
\hline
\end{tabular}

Tableau VI. Comparaison des vitesses de croissance des deux densités de la floraison à la récolte (croissance de la plante entière, du 13 août au 2 octobre).

\begin{tabular}{lccr}
$\begin{array}{l}\text { Plante entière } \\
\text { (jours 227, 234, 241, }\end{array}$ & $\begin{array}{l}\text { Critère de } \\
\text { comparaison }\end{array}$ & $\begin{array}{l}\text { Degrés } \\
\text { de liberté }\end{array}$ & Fà $1 \%$ \\
& & & \\
\hline & & & \\
Effet densité & 6,45 & $\mathrm{~F}(8,80)$ & 5,00 \\
Effet date & 29,68 & $\mathrm{~F}(14,80)$ & 3,14 \\
Interaction & 0,52 & $\mathrm{~F}(7,80)$ & 5,78
\end{tabular}


action entre date et densité, ce qui signifie que l'effet densité ne s'accentue pas (Tableau VI).

\section{Efficience de la transformation du rayonnement solaire en matière sèche (Tableau V)}

L'hypothèse de même évolution de l'indice foliaire pour les 2 traitements est confirmée par les notations de feuilles encore vertes et par l'arrêt de croissance des 2 couverts à la même date. Cela permet de comparer les rayonnements captés et les efficiences de croissance des 2 traitements.

Les efficiences sont analogues, ce qui permet de considérer que les 2 couverts ont des fonctionnements comparables pendant cette période.

\section{Production par organe}

- Production de grains et composantes du rendement. Les grains, dont la croissance commence longtemps après l'éclaircissage, ne sont affectés ni dans leur poids final par épi (Tableau VII), ni dans leur croissance (Fig. 1a).

Les nombres d'étages de grains ne sont ni différents entre traitements (Tableau VII), ni différents des nombres potentiels (Tableau IV); le nombre potentiel d'étages de grains a donc pu être limitant pour les 2 densités $d$ et é. Les nombres de grains par épi et les poids de 1000 grains ne sont pas différents, mais les nombres d'épis par plante le sont (Tableau VII).

A titre de comparaison, le traitement témoin $D$ (à forte densité pendant tout le cycle) présente un nombre d'étages plus faible que le nombre potentiel $(26,98$, écart-type $=2,85)$. Le poids de 1000 grains est significativement plus faible $(181,1 \mathrm{~g}$, écart-type $=32,27)$ que dans les traitements d et é.
- Poids et morphologie des tiges. Les tiges présentent, aux irrégularités d'échantillonnage près, les mêmes variations entre les deux traitements (Fig. 1b) : même date de début de décroissance (4 septembre), même quantité de réserves utilisées (différence entre le poids maximal et celui de la récolte : 16,1 et $14,84 \mathrm{~g} /$ plante respectivement pour $d$ et é), alors que les poids à l'éclaircissage sont sensiblement différents.

Sur l'ensemble de la période floraison-récolte (où les valeurs restent à leur maximum), les diamètres au collet sont significativement différents, tandis que les hauteurs ne le sont pas (Tableau VII). Notons que le poids à la récolte est inférieur au poids à la floraison pour les 2 traitements (Fig. 1b), mais il n'est inférieur au poids à l'éclaircissage que pour la faible densité (Fig. 1b).

\section{Discussion}

Le traitement "forte densité éclaircie» a permis de constituer un couvert où le rayonnement disponible par plante est proche de celui du couvert témoin avec un nombre potentiel de grains (observé environ 10 jours avant l'éclaircissage) comparable.

Les différences essentielles entre les deux couverts à l'éclaircissage sont le poids total par plante, la vitesse de croissance de l'ensemble de l'épi et le diamètre au collet.

Seule la croissance avant l'éclaircissage a été modifiée, la différenciation florale ne l'a pas été de façon sensible.

Effet de la densité de culture sur la différenciation des grains

L'absence d'effet de la densité sur le nombre potentiel de grains (déjà observé par Edmeades

Tableau VII. Caractéristiques du couvert à la récolte.

\begin{tabular}{|c|c|c|c|c|c|c|c|c|c|}
\hline \multirow[t]{2}{*}{ Récolte } & \multirow[t]{2}{*}{ Date } & \multicolumn{2}{|c|}{ Densité $d$} & \multicolumn{2}{|c|}{ Densité é } & \multirow{2}{*}{$\begin{array}{l}\text { Nb de } \\
\text { mesures }\end{array}$} & \multicolumn{3}{|c|}{$t$ à $5 \%$} \\
\hline & & moy & e. $-t$. & moy & e. $-t$. & & critère de & compa & aison \\
\hline Matière sèche $(\mathrm{g})$ & $22 / 10$ & & & & & & & & \\
\hline plante entière & & 207,5 & 34,7 & 190,8 & 22,5 & 18 & 1,71 & 2,11 & NS \\
\hline tige & & 50,2 & 10,2 & 43 & 6,4 & 18 & 2,54 & 2,11 & $\mathrm{~S}$ \\
\hline grains & & 100,8 & 15,8 & 97,7 & 10,5 & 18 & 0,69 & 2,11 & NS \\
\hline Nombre d'étages de & & & & & & & & & \\
\hline $\begin{array}{l}\text { grains } \\
\text { Composantes du rendement }\end{array}$ & $(2-9-22 / 10)$ & 30,57 & 2,34 & 30,07 & 1,92 & 54 & 1,21 & 2 & NS \\
\hline nb de grains/épi & $22 / 10$ & 410 & 54,2 & 399,3 & 37,5 & 18 & 0,69 & 2,11 & NS \\
\hline nb d'épis/plte & $11 / 9$ & 0,95 & 0,08 & 0,88 & 0,07 & 50 & 4,66 & 2,01 & $S$ \\
\hline poids de $1000 \mathrm{gr}$ & $22 / 10$ & 246,2 & 30,0 & 245,3 & 24,7 & 18 & 0,10 & 2,11 & NS \\
\hline Morphologie de la tige & & & & & & & & & \\
\hline hauteur (m) & 7 dates & 1,863 & 0,087 & 1,851 & 0,082 & 126 & 1,13 & 1,98 & NS \\
\hline $\begin{array}{l}\text { diamètre au collet } \\
\left(10^{-3} \mathrm{~m}\right)\end{array}$ & id. & 22,9 & 1,9 & 19,9 & 1,8 & 126 & 12,87 & 1,98 & $S$ \\
\hline
\end{tabular}




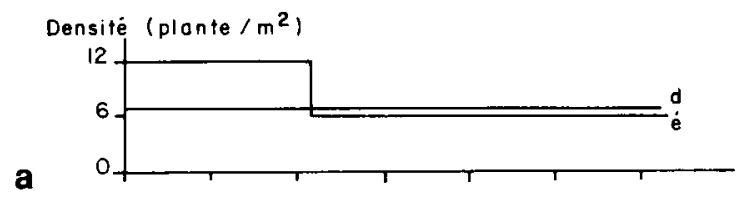

g / plonte $(\%)$

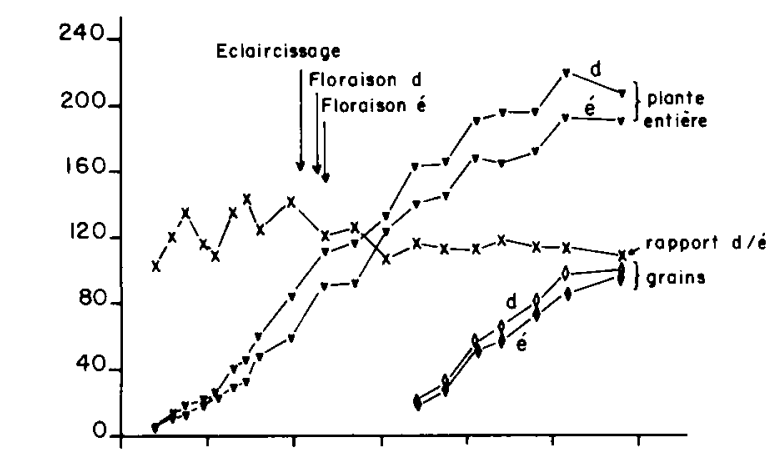

b g/plante Eeloircissoge

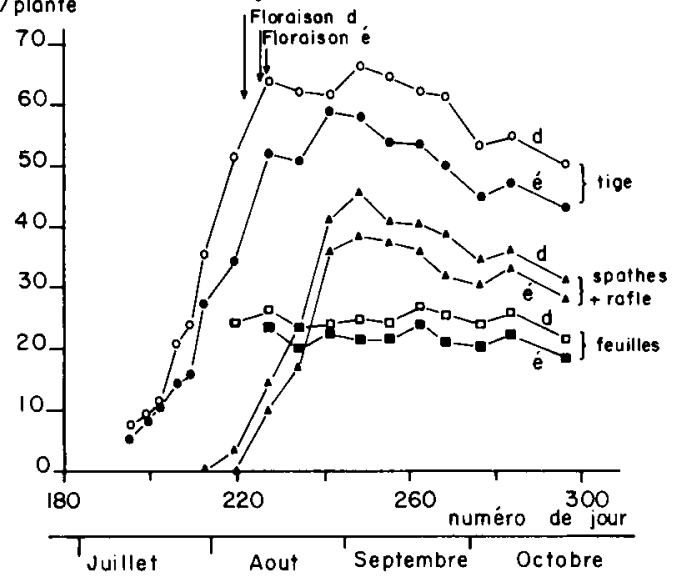

Fig. 1. Evolution de la matière sèche par plante pour les 2 traitements $(d=67500$ plantes/ha et é $=120000$ puis 60000 plantes/ha). Eclaircissage le 7 août (jour 220), floraison les 11 et 13 août (jours 224 et 226) : a. plante entière; grains; rapport des poids de matière sèche de plante entière entre les 2 traitements (d/é); b. tige; spathes + rafle; feuilles.

et Daynard (1979a) sur le nombre de fleurs initiées) est à souligner; il a été très utile ici dans la comparaison des fonctionnements des deux couverts après éclaircissage. II montre simplement que la forte densité de culture choisie (120 000 plantes/ha) est insuffisante pour que s'installe une compétition assez précoce entre plantes. En effet, la comparaison des matières sèches par plante dans les 2 traitements montre que la concurrence n'est sensible que tardivement (premières valeurs significativement différentes le 6 août, tandis que la différenciation est finie).

Croissance et répartition des assimilats de l'éclaircissage à la récolte

Rappelons que la différence de production acquise à l'éclaircissage se retrouve à la récolte, sur la tige et sur la partie non-grain de l'épi (spathes et rafle). Les grains ne sont pas affectés. Cela signifie que la différence d'état des couverts à l'éclaircissage n'a eu de conséquences ni sur la croissance ultérieure, ni sur la répartition des assimilats entre organes. Ces observations confirment la remarque de Tollenaar (1977) sur l'absence de concurrence entre tige et épi.

Ces résultats montrent que les relations entre un indicateur choisi à la floraison et la production finale s'appuient sur la persistance d'un certain niveau de rayonnement disponible par plante : dans notre expérimentation le niveau de rayonnement disponible par plante a été modifié par l'éclaircissage et le classement des productions à la floraison ne se répercute pas sur la production de grains : l'indicateur a perdu sa valeur de prédiction.

Dans le traitement $D$, le rayonnement disponible par plante est plus faible. La production de grains est plus faible : le niveau de rayonnement disponible après l'éclaircissage a été limitant. Comme nombre de grains et poids des grains sont affectés, on peut conclure que le rayonnement a été limitant aux périodes où se fixaient ces 2 composantes c'est-à-dire pendant l'ensemble de la période.

Ce résultat concorde avec celui de Edmeades et Daynard (1979b) : ils démontrent, sur un couvert de plantes hétérogènes, que les niveaux de photosynthèse mesurés à la floraison constituent un bon indicateur de la production de grains par plante. Ils concluent que de telles relations ne prouvent pas l'existence d'un phénomène crucial à la date choisie, mais reflètent l'importance du régime radiatif établi entre plantes, lié dans leur cas à l'hétérogénéité entre plantes du couvert. II s'agit donc ici d'un effet de la répartition du rayonnement entre plantes, donc des surfaces foliaires relatives de chaque plante.

Si un indicateur comme celui de Edmeades et Daynard (la photosynthèse dans les 10 jours qui suivent la floraison) est utilisé dans d'autres conditions, par exemple dans des comparaisons entre conditions climatiques (lieux ou années), il privilégiera l'autre facteur essentiel de la photosynthèse, le rayonnement climatique disponible, alors que les effets démontrés sont ceux des effets de répartition du rayonnement entre plantes par Edmeades et Daynard et du niveau de rayonnement disponible par plante pendant l'ensemble du remplissage, dans notre cas.

\section{Niveau d'utilisation des réserves}

Les poids de 1000 grains finaux égaux entre les 2 cultures montrent que la qualité de remplissage des grains est la même. Le fait que les réserves utilisées soient les mêmes pour les 2 cultures prouve alors que ni les réserves, ni les capacités de réserves n'ont été limitantes. En effet, les 
grains ont été remplis de la même façon, en partie sur des réserves. Celles-ci, bien que constituées par des plantes à poids et volume de tige différents, ont été utilisées en même quantité. La mise en réserve a donc été prioritaire sur la croissance de la tige et la quantité de réserves faites non limitée par le volume de la tige.

Le poids final de tiges inférieur au poids à l'éclaircissage en faible densité montre, dans ce cas, la constitution très précoce de réserves, avant que la tige ait atteint sa taille finale, ce qui n'est pas le cas pour la culture à forte densité éclaircie.

Dans le poids total de grains, la part de réserves est, au minimum, la différence entre croissances des grains et de la plante entière pendant le remplissage des grains. Elle s'élève à 25,7 et $31,3 \%$ respectivement pour $d$ et é.

Les réserves de la tige sont utilisées jusqu'à 24,3 et $27,0 \%$ du poids maximal de matière sèche des tiges respectivement pour $d$ et é. Cette utilisation de réserves correspond bien à celle étudiée par d'autres auteurs (Heuer, 1982; Daynard et al., 1969) et à la modélisation qui en est faite (modèle CERES de Jones et Kiniry, 1986).

Le fonctionnement des 2 cultures peut donc se schématiser de la façon suivante : dans la phase antérieure à l'éclaircissage, où les disponibilités en assimilats par plante sont très différentes, les biomasses totales fabriquées sont significativement différentes, sans que les nombres de fleurs, ni les nombres de grains potentiels ne soient affectés. Dans la phase postérieure à l'éclaircissage, où les disponibilités en assimilats sont du même ordre de grandeur, les grains sont remplis de la même façon pour les 2 cultures : seul, le rayonnement disponible détermine alors la proportion de grains remplis parmi les grains potentiels (tels qu'ils sont définis par le gradient de différenciation le long de l'épi).

Les seules grandeurs significativement différentes à la récolte sont le poids final de tige et son diamètre au collet pour la plante et le nombre moyen d'épis par plante pour le couvert. Ces grandeurs sont toutes définies dans une période proche de l'éclaircissage. La privation d'assimilats provoquée par la forte densité n'aurait donc que des effets immédiats : cette observation est à confirmer dans d'autres conditions.

\section{Conclusion}

La conclusion essentielle est que, pour deux couverts ne se distinguant que par le rayonnement disponible par plante avant floraison, ce qui est le cas avec l'éclaircissage, et sans que le nombre de grains potentiel avant et après floraison soit affecté, la production de grains dépend seulement du rayonnement disponible par plante après floraison. II est donc possible que la production globale de la plante soit affectée par ses conditions de croissance dans les stades antérieurs à la floraison, sans que cela ait de conséquences sur la production de grains.

La comparaison avec la culture à forte densité pendant l'ensemble du cycle apporte confirmation de l'effet du niveau de rayonnement disponible pendant l'ensemble du remplissage aussi bien sur le nombre que sur le remplissage des grains.

Bien que les tiges aient des poids finaux différents, les mêmes quantités de réserves sont utilisées. On peut en conclure que les réserves ne sont affectées :

- ni dans leur constitution - au moins pour la part qui en est utilisée,

- ni dans leurs capacités de stockage - malgré les tailles différentes de tiges,

- ni dans leur utilisation.

Pour des cultures en densité double de la normale, la différence de productivité en grains n'est pas encore acquise de façon irréversible avant la floraison : une augmentation de la disponibilité en assimilats à cette date permet d'atteindre une production de grains par plante analogue, bien que la production globale, en particulier celle de la tige, soit, elle, définitivement affectée.

Enfin, ces résultats semblent avoir un intérêt essentiel dans les modèles de production de couvert, puisqu'il apparaît clairement

- que les réserves et leur niveau d'utilisation jouent un rôle essentiel dans le remplissage des grains,

- que la répartition des assimilats entre plantes dépend largement de la densité de culture alors que celle-ci n'est prise en compte que de façon multiplicative, même dans des modèles aussi élaborés que CERES.

Ces conclusions méritent d'être confirmées, en particulier pour d'autres lieux-années et d'autres densités de culture où pourraient apparaître des effets plus précoces de compétition entre plantes : il est probable que de telles conditions puissent affecter de façon plus durable, donc plus marquée, le fonctionnement du couvert.

La comparaison de génotypes de précocités différentes modifie, elle, la position de la période de remplissage par rapport aux conditions climatiques, donc les conditions de remplissage ellesmêmes. Elle sera traitée dans un travail ultérieur. 


\section{Références}

Bonhomme R., Ruget F., Derieux M. \& Vincourt P. (1982) Relations entre production de matière sèche aérienne et énergie interceptée chez différents génotypes de maïs. C.R. Acad. Sci., série D, 294, 393-398

Daynard T.B., Tanner J.W. \& Hume D.J. (1969) Contribution of stalk soluble carbohydrates to grain yield in corn (Zea mays L.). Crop Sci. 9, 831-834

Derieux M., Bonhomme R., Duburcq J.-B. \& Ruget F. (1985) Variations du nombre de grains chez différents génotypes de maïs Agronomie 5, (4), 355-359

Duncan W.G. (1975) Maize. In : Crop Physiology. L.T. Evans, 1975, 23-50

Edmeades G.O. \& Daynard T.B. (1979a) The development of plant-to-plant variability in maize at different planting densities. Can. J. Plant Sci. 59, 561-576

Edmeades G.O. \& Daynard T.B. (1979b) The relationship between final yield and photosynthesis at flowering in individual maize plants. Can. J. Plant. Sci. 59, 585-601

Fuchs A. (1977) Der Einfluss der organogenetischen Entwicklung auf die Ertragsbildung von F1-Hybriden des Maises und deren Eltern. Arch. Züchtungsforsch. Berlin 7, 3, 149-157

Hanway J.J. \& Russel W.A. (1969) Dry matter accumu- lation in corn (Zea mays L.) plants: comparisons among single cross hybrids. Agr. J. 61, 6, 947-951

Hawkins R.C. \& Cooper P.J.M. (1981) Growth, development and grain yield of maize. Expl. Agric. 17, 203207

Heuer M.L. (1982) Response of maize to several moisture stress treatments as determined by growth analyses. Thesis Master of Science, lowa State University, Ames, lowa

Jones C.A. \& Kiniry J.R. (1986) CERES-maize, a simulation model of maize growth and development, Texas A \& M University

Jones R.J. \& Simmons S.R. (1983) Effect of altered source-sink ratio on growth of maize kernels. Crop Sci. 23, 1, 129-134

Landi P. \& Concilio L. (1982) Analisi del ritmo di estrusione degli stili in ibridi di mais allevati a diversi levelli di densità di piante. Riv. Agron. 16, 1, 297-300

Ruget F. (1987) Evaluation du nombre de grains potentiels à la récolte à partir d'observations de développement floral antérieur à la floraison, chez le maïs. C.R.Acad. Sc. Paris, série III, 304, 185-190

Tollenaar M. (1977) Sink-source relationships during reproductive development in maize. A review. Maydica XXII, 49-75 